\title{
Eco-geographic Distribution and Microcenters of Genetic Diversity in Faba Bean (Vicia Faba L.) and Field Pea (Pisum Sativum L.) Germplasm Collections from Ethiopia
}

\author{
Gemechu Keneni*, Mussa Jarso and Tezera Wolabu \\ Holetta Agricultural Research Center, P O Box 2003, Addis Ababa, Ethiopia
}

\begin{abstract}
Ethiopia is considered an important center of secondary diversity for both faba bean (Vicia faba L.) and field pea (Pisum sativum L.). However, areas of eco-geographic distribution and the microcenter of genetic diversity are not well known. Two separate trials consisting of 160 faba bean and 148 field pea accessions were conducted at Holetta and Kulumsa in 2001. Simple and alpha-lattice designs with 2 replications were used for faba bean and field pea, respectively. Cluster analysis grouped faba bean accessions into eight and field pea into five different classes. Mahalanobis's $\mathrm{D}^{2}$ analyses showed significant genetic distances between most of the clusters in both crops. Accessions from the northern parts of the country showed tendencies of being grouped together, indicating that their genetic background may be related. Accessions from the southern parts of the country were distributed over most of the clusters showing more genetic diversity compared to those from the northern parts. This indicates that the distribution of genetic diversity among accessions is not uniform across different eco-geographical regions in Ethiopia and the microcenter of genetic diversity for both crops may be located in the southern part of the country. The existence of more genetic diversity in one region compared to the other could be due to differences in the level of variability among the original introductions to different regions, the nature and degree of both human and natural selections after introduction, and effects of ecological and agricultural conditions as major forces of evolution. Future collection, conservation and utilization programs should focus on the southern part to safeguard and exploit the tremendous genetic diversity. However, a comprehensive study involving both morpho-agronomic traits and molecular markers would be needed for a more comprehensive conclusion.
\end{abstract}

Key words: Cluster Analysis; Eco-geographic Distribution; Faba bean (Vicia faba L.); Field pea (Pisum sativum L.); Genetic Diversity; Mahalanobis's Distance $\left(\mathrm{D}^{2}\right)$; Microcenter of Genetic Diversity

\section{Introduction}

Agricultural biodiversity, comprising all the elements from gene to agricultural ecosystems, is one of the principal components of natural resources (Ford-Lloyd and Jackson, 1986; Chahal and Gosal, 2002; Singh, 2002; Atta-Krah, 2004), even though it is often treated separately from other natural resources in many parts of the world (Atta-Krah, 2004), including Ethiopia. A reservoir of irreplaceable genes and gene complexes of a number of crops is currently being lost at a rapid rate through genetic erosion as a result of displacement of landraces by modern varieties, dynamics of agriculture and land uses, destruction of natural habitats, and drought (Tesema and Eshetayehu, 2004). Genetic erosion, the gradual depletion of natural resources in general and crop germplasm in particular with both natural and artificial interferences is, therefore, a current topic all over the world. The conservation and management of germplasm, like that of other components of natural resources, is very crucial not only for food security but also for sustainable agriculture and environmental health.

The Mediterranean and Central Asian regions are regarded as the probable centers of origin and domestication of faba bean (Vicia faba L.) and field pea (Pisum sativum L.) (Westphal, 1974; Davies, 1976; Hagedorn, 1984; Chahal and Gosal, 2002; Singh, 2002). It is assumed that the crops were brought to Ethiopia directly from Central Asia immediately after domestication (Asfaw et al., 1994 a,b; Yohannes , 2000) and have been grown since antiquity (Dawit et al., 1994). Ethiopia is, undoubtedly, considered as one of the important centers of secondary diversity for both faba bean (Asfaw et al., 1994 a,b; Yohannes , 2000; Singh, 2002) and field pea (Hagedorn, 1984; Hailu et al., 1991; Singh, 2002). Wild and primitive forms of field pea are also known to exist in the high elevations of Ethiopia (Hagedorn, 1984), and hence, some authorities including Vavilov (1951) considered Ethiopia rather as one of the primary centers of diversity (Singh, 2002; FAO, 1998). Ethiopia is a country of great eco-geographical diversity with high and rugged mountains (EMA, 1988). This ecogeographic diversity might have resulted in diverse crop germplasm in general (Vavilov, 1951; Singh, 2002), including those of faba bean and field pea in particular (Vavilov, 1951; Harlan, 1969). 
Reports exist both from Ethiopia and elsewhere in different crop species (Ford-Lloyd and Jackson, 1986; Demissie and Bj申rnstad, 1997; Nigussie , 2001; Chahal and Gosal, 2002; Singh, 2002) including faba bean and field pea (Singh and Tripathi, 1985; Hailu et al., 1991; Dawit et al., 1994) that diversity in germplasm may not be uniformly distributed across eco-geographic regions but rather concentrated in a particular region depending on ecological, agricultural and socio-cultural conditions in the area (Chahal and Gosal, 2002; Singh, 2002). According to Singh (2002), small areas within the large center of diversity, called microcenters, may exhibit a much greater diversity than the center as a whole. The determination of the eco-geographic distribution and microcenters with higher genetic diversity is rewarding in terms of effective germplasm collection, conservation and utilization programs (Bartual et al., 1985; Dale et al., 1985; Ford-Lloyd and Jackson, 1986; Rezai and Frey, 1990; Jaradat, 1991; Demissie and Bjфrnstad, 1997; Chahal and Gosal, 2002; Singh, 2002). Such information is also helpful to locate sites for in-situ conservation and those for crop evolution studies (Singh, 2002).
Even though it is generally believed that the Ethiopian faba bean and field pea landraces have valuable genetic diversity (Harlan, 1969; Van der Maesen et al., 1988; Engels and Hawkes, 1991; Gemechu, K. et al., 2003 a,b), the eco-geographic distribution and microcenters of genetic diversity are not yet well known. This experiment was, therefore, designed to generate information on the eco-geographic distribution and microcenter of genetic diversity for faba bean and field pea germplasm in Ethiopia.

\section{Materials and Methods}

Accessions of 160 faba bean and 148 field pea collected from the most important faba bean and field pea producing eco-geographic regions of Ethiopia (Ethiopian Agricultural Sample Enumeration, 2002) varying in altitude, rainfall, temperature and soil type were considered for the study in two separate trials. They were collected from the most important production complexes in Wello, Gonder, Shewa and Arsi (Figure 1). The accessions included in the study from each region are given in Tables $1 \mathrm{a}$ and $\mathrm{b}$.
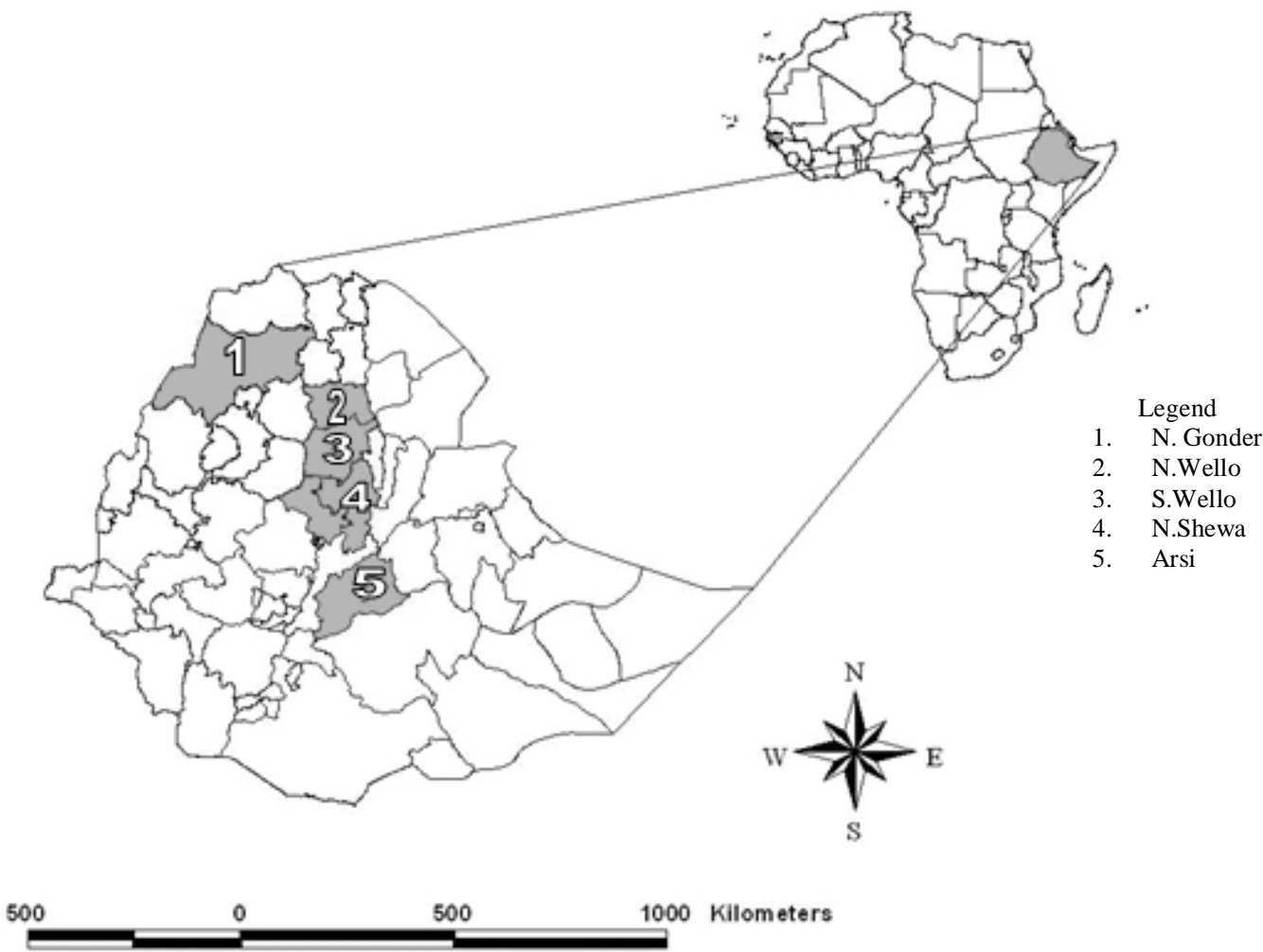

Figure 1. Map of Ethiopia showing origins of faba bean and field pea accessions considered in the study 
Table 1a. Origin and designations of the test faba bean accessions

\begin{tabular}{llcll}
\hline $\begin{array}{l}\text { Geographic } \\
\text { origin }\end{array}$ & Districts of origin & $\begin{array}{l}\text { No. of } \\
\text { collections } \\
\text { evaluated }\end{array}$ & \\
\hline Wello & Wadla, Delanta, Wagal Tena, & 29 & FBColl-1/99, FBColl-2/99, FBColl-3/99, FBColl-4/99, FBColl-5/99, FBColl-6/99, FBColl-7/99, \\
& Ambassel, Kutaber, Tenta & & $\begin{array}{l}\text { FBColl-8/99, FBColl-9/99, FBColl-10/99, FBColl-11/99, FBColl-12/99, FBColl-13/99, FBColl- } \\
\end{array}$ \\
& & 14/99, FBColl-15/99,FBColl-16/99, FBColl-17/99, FBColl-18/99, FBColl-19/99, FBColl-20/99, \\
& & FBColl-21/99, FBColl-22/99, FBColl-23/99, FBColl-24/99, FBColl-25/99, FBColl-26/99, FBColl- \\
& $27 / 99$, FBColl-28/99, FBColl-29/99
\end{tabular}

Gonder Gonder Zuria, Lay Armacho, Wogera, Dabat and Dabark
40

FBColl-58/99, FBColl-59/99, FBColl-60/99, FBColl-61/99, FBColl-62/99, FBColl-63/99, FBColl64/99, FBColl-65/99, FBColl-66/99, FBColl-67/99, FBColl-68/99, FBColl-69/99, FBColl-70/99, FBColl-71/99, FBColl-72/99, FBColl-73/99, FBColl-74/99, FBColl-75/99, FBColl-76/99, FBColl77/99, FBColl-78/99, FBColl-79/99, FBColl-80/99, FBColl-81/99, FBColl-82/99, FBColl-83/99, FBColl-84/99, FBColl-85/99, FBColl-86/99, FBColl-87/99, FBColl-88/99, FBColl-89/99, FBColl90/99, FBColl-91/99, FBColl-92/99, FBColl-93/99, FBColl-94/99, FBColl-95/99, FBColl-96/99, FBColl-97/99
Shewa Basona Warana, Tarmaber, Lalomama/Molale, Lalo Mider, Mehal Meda, Gera Keya and Sela Dingay
41

FBColl-133/99, FBColl-134/99, FBColl-135/99, FBColl-136/99, FBColl-137/99, FBColl-138/99, FBColl-139/99, FBColl-140/99, FBColl-141/99, FBColl-142/99, FBColl-143/99, FBColl-144/99, FBColl-145/99, FBColl-146/99, FBColl-147/99, FBColl-148/99, FBColl-149/99, FBColl-150/99, FBColl-151/99, FBColl-152/99, FBColl-153/99, FBColl-154/99, FBColl-155/99, FBColl-156/99, FBColl-157/99, FBColl-158/99, FBColl-159/99, FBColl-160/99, FBColl-161/99, FBColl-162/99, FBColl-163/99, FBColl-164/99, FBColl-165/99, FBColl-166/99, FBColl-167/99, FBColl-168/99, FBColl-169/99, FBColl-170/99, FBColl-171/99, FBColl-172/99, FBColl-173/99
Tiyo \& Digelu, Lemu \& Bilbilo, Shirka, Gedeb Asasa, Kofele, Munesa, Hetosa, Tena, Robe, Amigna, Seru, Sude, Dodota Sire, Jeju, Merti and Chole

\begin{tabular}{ll}
\hline Arsi & Tiyo \& Digelu, Lemu \& \\
& Bilbilo, Shirka, Gedeb Asasa, \\
& Kofele, Munesa, Hetosa, \\
& Tena, Robe, Amigna, Seru, \\
& Sude, Dodota Sire, Jeju, \\
& Merti and Chole
\end{tabular}

50

FBColl-157/00, FBColl-159/00, FBColl-161/00, FBColl-163/00, FBColl-164/00, FBColl-168/00, FBColl-170/00, FBColl-172/00, FBColl-176/00, FBColl-178/00, FBColl-181/00, FBColl-184/00, FBColl-187/00, FBColl-188/00, FBColl-191/00, FBColl-193/00, FBColl-196/00, FBColl-199/00, FBColl-201/99, FBColl-204/00, FBColl-205/00, FBColl-208/00, FBColl-209/00, FBColl-211/00, FBColl-214/00, FBColl-217/00, FBColl-220/00, FBColl-222/00, FBColl-224/00, FBColl-226/00, FBColl-228/00, FBColl-231/00, FBColl-233/00, FBColl-236/00, FBColl-238/00, FBColl-240/00, FBColl-242/00, FBColl-244/00, FBColl-246/00, FBColl-248/00, FBColl-250/00, FBColl-252/00, FBColl-255/00, FBColl-257/00, FBColl-259/00, FBColl-261/00, FBColl-263/00, FBColl-265/00, FBColl-267/00, FBColl-269/00 
Table $1 \mathrm{~b}$. Origin and designations of the test field pea accessions

\begin{tabular}{|c|c|c|c|}
\hline $\begin{array}{l}\text { Geographic } \\
\text { origin }\end{array}$ & Districts of origin & $\begin{array}{l}\text { No. of } \\
\text { collections } \\
\text { evaluated }\end{array}$ & Designations \\
\hline Wello & $\begin{array}{l}\text { Wadla, Delanta, Wagal Tena, Ambassel, } \\
\text { Kutaber and Tenta }\end{array}$ & 26 & $\begin{array}{l}\text { FPColl-30/99, FPColl-31/99, FPColl-32/99, FPColl-33/99, FPColl-34/99, FPColl- } \\
\text { 35/99, FPColl-36/99, FPColl-37/99, FPColl-38/99, FPColl-39/99, FPColl-40/99, } \\
\text { FPColl-41/99, FPColl-42/99, FPColl-43/99, FPColl-44/99, FPColl-45/99, FPColl- } \\
\text { 47/99, FPColl-48/99, FPColl-49/99, FPColl-50/99, FPColl-51/99, FPColl-52/99, } \\
\text { FPColl-53/99, FPColl-54/99, FPColl-55/99, FPColl-57/99 }\end{array}$ \\
\hline Gonder & $\begin{array}{l}\text { Gonder Zuria, Lay Armacho, Wogera, } \\
\text { Dabat and Dabark }\end{array}$ & 31 & $\begin{array}{lllll}\text { FPColl-98/99, } & \text { FPColl-99/99, } & \text { FPColl-100/99, } & \text { FPColl-101/99, } & \text { FPColl-102/99, } \\
\text { FPColl-103/99, } & \text { FPColl-104/99, } & \text { FPColl-105/99, } & \text { FPColl-106/99, } & \text { FPColl-107/99, } \\
\text { FPColl-108/99, } & \text { FPColl-110/99, } & \text { FPColl-111/99, } & \text { FPColl-112/99, } & \text { FPColl-114/99, } \\
\text { FPColl-115/99, } & \text { FPColl-116/99, } & \text { FPColl-117/99, } & \text { FPColl-119/99, } & \text { FPColl-120/99, } \\
\text { FPColl-121/99, } & \text { FPColl-122/99, } & \text { FPColl-123/99, } & \text { FPColl-125/99, } & \text { FPColl-126/99, } \\
\text { FPColl-127/99, } & \text { FPColl-128/99, } & \text { FPColl-129/99, } & \text { FPColl-130/99, } & \text { FPColl-131/99, } \\
\text { FPColl-132/99 } & & & & \end{array}$ \\
\hline Shewa & $\begin{array}{l}\text { Basona Warana, } \\
\text { Lalomama/Molale, Mehal } \\
\text { Keya and Sela Dingay }\end{array}$ & 36 & $\begin{array}{lllll}\text { FPColl-182/99, } & \text { FPColl-183/99, } & \text { FPColl-184/99, } & \text { FPColl-185/99, } & \text { FPColl-186/99, } \\
\text { FPColl-187/99, } & \text { FPColl-188/99, } & \text { FPColl-189/99, } & \text { FPColl-190/99, } & \text { FPColl-191/99, } \\
\text { FPColl-192/99, } & \text { FPColl-193/99, } & \text { FPColl-194/99, } & \text { FPColl-195/99, } & \text { FPColl-196/99, } \\
\text { FPColl-197/99, } & \text { FPColl-198/99, } & \text { FPColl-199/99, } & \text { FPColl-200/99, } & \text { FPColl-201/99, } \\
\text { FPColl-202/99, } & \text { FPColl-203/99, } & \text { FPColl-204/99, } & \text { FPColl-205/99, } & \text { FPColl-206/99, } \\
\text { FPColl-207/99, } & \text { FPColl-208/99, } & \text { FPColl-209/99, } & \text { FPColl-210/99, } & \text { FPColl-211/99, } \\
\text { FPColl-212/99, } & \text { FPColl-213/99, } & \text { FPColl-214/99, } & \text { FPColl-215/99, } & \text { FPColl-216/99, } \\
\text { FPColl-217/99 } & & & \end{array}$ \\
\hline Arsi & $\begin{array}{l}\text { Digalu \& Tijo, Shirka, Lemu \& Bilbilo, } \\
\text { Gedeb Asasa, Kofele, Munesa, Hetosa, } \\
\text { Amigna, Seru, Robe and Tena }\end{array}$ & 55 & $\begin{array}{l}\text { FPColl-30/00, FPColl-31/00, FPColl-32/00, FPColl-33/00, FPColl-34/00, FPColl- } \\
\text { 35/00, FPColl-36/00, FPColl-37/00, FPColl-38/00, FPColl-39/00, FPColl-40/00, } \\
\text { FPColl-41/00, FPColl-42/00, FPColl-43/00, FPColl-44/00, FPColl-45/00, FPColl- } \\
\text { 46/00, FPColl-47/00, FPColl-48/00, FPColl-49/00, FPColl-50/00, FPColl-51/00, } \\
\text { FPColl-52/00, FPColl-53/00, FPColl-54/00, FPColl-55/00, FPColl-56/00, FPColl- } \\
57 / 00, \text { FPColl-58/00, FPColl-59/00, FPColl-60/00, FPColl-61/00, FPColl-62/00, } \\
\text { FPColl-63/99, FPColl-64/00, FPColl-65/00, FPColl-66/00, FPColl-67/00, FPColl- } \\
68 / 00 \text {, FPColl-69/00, FPColl-70/00, FPColl-71/00, FPColl-72/00, FPColl-73/00, } \\
\text { FPColl-74/00, FPColl-75/00, FPColl-76/00, FPColl-77/00, FPColl-78/00, FPColl- } \\
\text { 79/00, FPColl-80/00, FPColl-81/00, FPColl-82/00, FPColl-83/00, FPColl-84/00 }\end{array}$ \\
\hline
\end{tabular}


The accessions were evaluated at two locations, Holetta $\left(09^{\circ} 00^{\prime} \mathrm{N}, 38^{\circ} 30^{\prime} \mathrm{E}\right)$ and Kulumsa $\left(08^{\circ} 01^{\prime} \mathrm{N}, 39^{\circ} 09^{\prime} \mathrm{E}\right)$ in Ethiopia during the 2001 main season in a separate trial for each crop. Holetta, with an altitude of 2400 meters above sea level and average annual rainfall of $1000 \mathrm{~mm}$, represents the major high-altitude production areas of the country, while Kulumsa, with an altitude of 2200 meters above sea level and average annual rainfall of $800 \mathrm{~mm}$, represents the major mid-altitude production areas. Holetta is characterized by a redbrown clay soils with a $\mathrm{pH}$ below 5 and Kulumsa by a dark-clay loam soil with a $\mathrm{pH}$ of 6.0. The trials were laid down in simple and alpha-lattice designs with two replications for faba bean and field pea, respectively. Seeds were planted on plots consisting of two rows of $4 \mathrm{~m}$ length with a spacing of $40 \mathrm{~cm}$ for faba bean and $20 \mathrm{~cm}$ for field pea between rows and $5 \mathrm{~cm}$ between plants within a row for both crops. Fertilizer was applied at the rate of $46 \mathrm{~kg} \mathrm{ha}^{-1} \mathrm{P}_{2} \mathrm{O}_{5}$ and $18 \mathrm{~kg} \mathrm{~N} \mathrm{ha}^{-1}$ while management practices followed research recommendations specific to each location. Data were collected on days to flowering, days to maturity, grain filling period (days to maturity minus days to flowering), plant height $(\mathrm{cm})$, chocolate spot (Botrytis fabae Sard.) infestation level in faba bean and Ascochyta blight (Mycosphaerella pinodes Lib.) in field pea, number of nodes/plant, number of podding nodes/plant, number of pods/podding nodes, number of pods/plant, number of seeds/pod, 1000 seed weight $(\mathrm{g})$, and grain yield/plot (g). Records on both chocolate spot and aschochyta blight were taken based on 1-9 scale, 1 being highly resistant and 9 highly susceptible. Records on diseases scores were pre-transformed to percentage values, which then ARCSINE transformed for statistical analysis as suggested by Little and Hills (1978). Data on all traits were pre-standardized to means of zero and variances of unity before clustering to avoid bias due to differences in measurement scales (Manly, 1986).

Clustering of accessions was performed by average linkage method of SAS software (SAS Institute, 1996) using traits that were found to be significantly different among the accessions at least at one of the locations. Points where local peaks of the pseudo F statistic join with small values of the pseudo $t^{2}$ statistic followed by a larger pseudo $t^{2}$ for the next cluster fusion were examined to decide the number of clusters (SAS Institute, 1996). Genetic distances between clusters as standardized Mahalanobis's $D^{2}$ statistics were calculated as: $\mathrm{D}_{\mathrm{ij}}^{2}=(\mathrm{xi}$ $x j)^{\prime} \operatorname{cov}^{-1}(x i-x j)$ Where, $D^{2} i j=$ the distance between cases $i$ and $j$; $x i$ and $x j=$ vectors of the values of the variables for cases $i$ and $j$; and $\operatorname{cov}^{-1}=$ the pooled within groups variance-covariance matrix.

The $\mathrm{D}^{2}$ values obtained for pairs of clusters were considered as the calculated values of Chi-square $\left(\chi^{2}\right)$ and were tested for significance both at $1 \%$ and $5 \%$ probability levels against the tabulated value of $\chi^{2}$ for ' $P$ ' degree of freedom, where $\mathrm{P}$ is the number of characters considered (Singh and Chaudhary, 1985).

\section{Results and Discussion}

\subsection{Performance of Accessions}

Separate analyses of variance across the two locations showed significant differences among the accessions while pooled differences were mostly non-significant as discussed elsewhere (Gemechu Keneni et al., 2005 a \& b). A stratified ranking diagram showed that accessions from different eco-geographical zones had more or less different pattern of distribution for the two most important economic traits (grain yield and seed size). The faba bean accessions collected from the southern parts of the country (Arsi) showed clear superiority as most of them ranked in the middle and top one-third for both traits. On the other hand, most of the faba bean collections from the northern parts of the country and those from Shewa ranked either in the bottom one-third or in the middle one-third. Performance among collections from different eco-geographical regions for grain yield and seed size was not distinct for field pea as it was for faba bean. A few accessions ranked in the top one-third not only from Arsi but also from Shewa for grain yield and Wello and Shewa for seed size while most others were either in the middle or bottom onethird (Figure 2).

Generally, collections from Arsi revealed more concentration of desirable genes not only in terms of grain yield and seed size in both crops but also those for better pod load and chocolate spot resistance in faba bean compared to collections from other parts of the country (data not shown).

\subsection{Extent and Pattern of Genetic Diversity}

Cluster analyses showed that accessions of faba bean were grouped into eight while that of field pea were grouped into five distinct clusters. The number of accessions varied from cluster to cluster. Some clusters had only a single accession while others had as high as 50 in faba bean and 66 in field pea (Tables $2 a$ and b). 


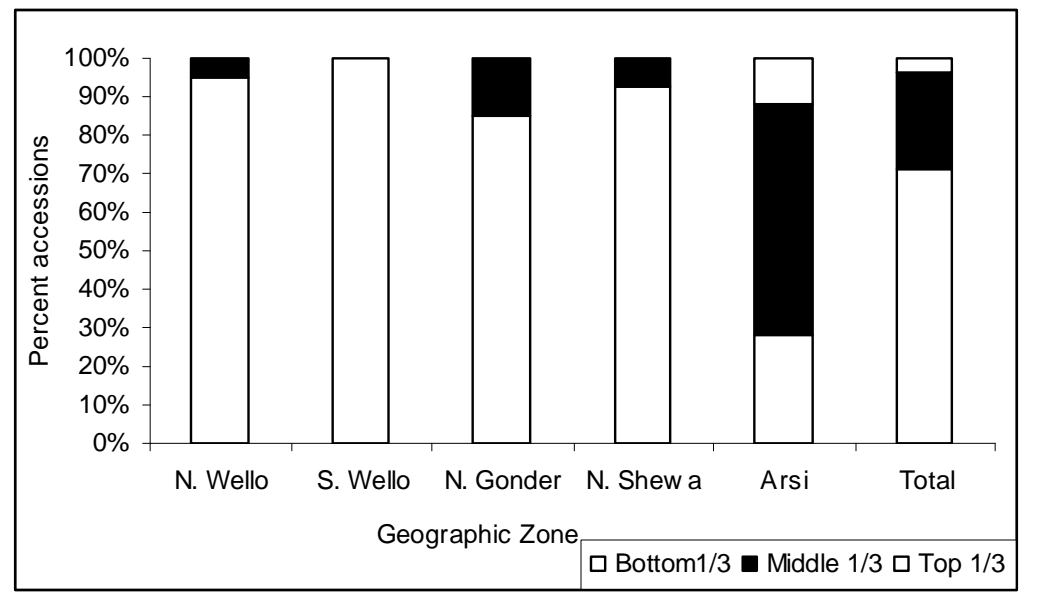

(A) Grain yield in faba bean

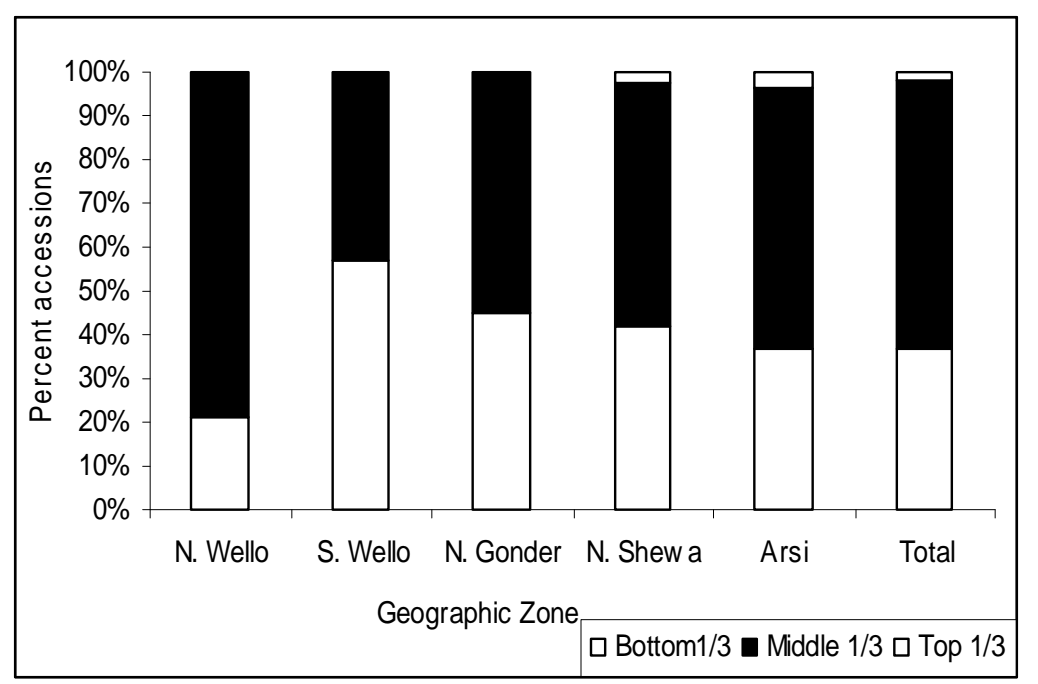

(C) Grain yield in field pea

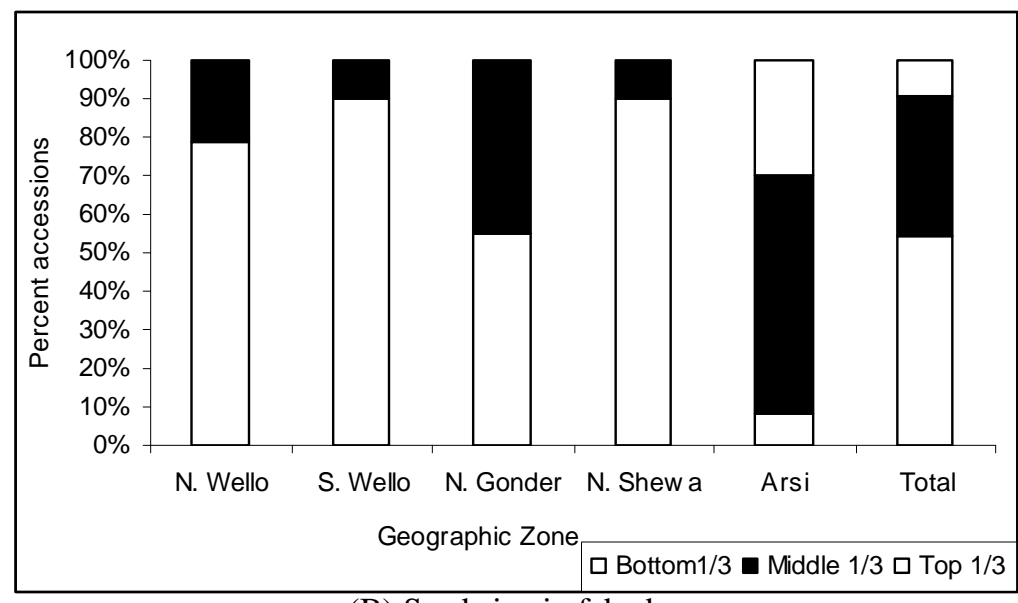

(B) Seed size in faba bean

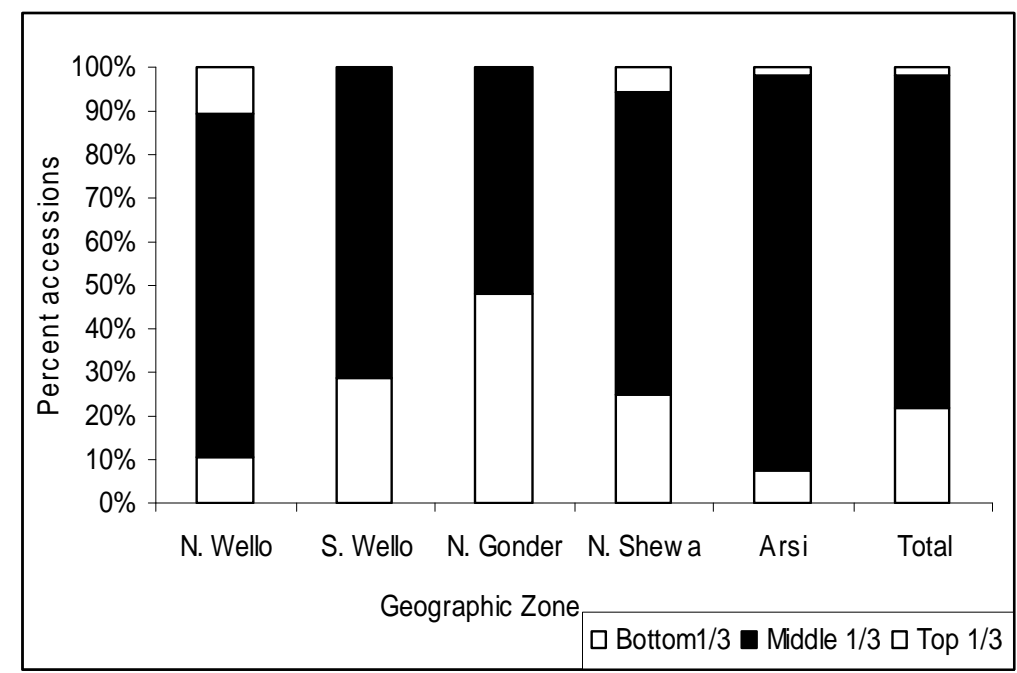

(D) Seed size in field pea

Figure 2. Stratified ranking diagram showing the distributions for two economic traits (grain yield and seed size) in 160 faba bean and 148 field pea accessions collected from different eco-geographical zones of Ethiopia 
Table 2a. Grouping of 160 faba bean accessions into different clusters

\begin{tabular}{|c|c|c|}
\hline Cluster & $\begin{array}{l}\text { No. of accessions } \\
\text { in cluster }\end{array}$ & Designations \\
\hline $\mathrm{C}_{1}$ & 30 & $\begin{array}{l}\text { FBColl-1/99, FBColl-2/99, FBColl-3/99, FBColl-4/99, FBColl-5/99, FBColl-7/99, FBColl-8/99, FBColl-9/99, } \\
\text { FBColl-10/99, FBColl-11/99, FBColl-12/99, FBColl-13/99, FBColl-14/99, FBColl-15/99, FBColl-16/99, FBColl- } \\
\text { 17/99, FBColl-18/99, FBColl-19/99, FBColl-20/99, FBColl-21/99, FBColl-22/99, FBColl-24/99, FBColl-25/99, } \\
\text { FBColl-27/99, FBColl-28/99, FBColl-136/99, FBColl-149/99, FBColl-154/99, FBColl-158/99, FBColl-166/99 }\end{array}$ \\
\hline $\mathrm{C}_{2}$ & 50 & $\begin{array}{l}\text { FBColl-73/99, FBColl-78/99, FBColl-75/99, FBColl-66/99, FBColl-79/99, FBColl-68/99, FBColl-71/99, FBColl- } \\
\text { 89/99, FBColl-83/99, FBColl-80/99, FBColl-85/99, FBColl-81/99, FBColl-91/99, FBColl-62/99, FBColl-92/99, } \\
\text { FBColl-93/99, FBColl-90/99, FBColl-69/99, FBColl-88/99, FBColl-64/99, FBColl-96/99, FBColl-84/99, FBColl- } \\
\text { 86/99, FBColl-82/99, FBColl-67/99, FBColl-77/99, FBColl-141/99, FBColl-155/99, FBColl-70/99, FBColl-72/99, } \\
\text { FBColl-76/99, FBColl-94/99, FBColl-97/99, FBColl-263/00, FBColl-61/99, FBColl-193/00, FBColl-196/00, FBColl- } \\
\text { 238/00, FBColl-240/00, FBColl-250/00, FBColl-252/00, FBColl-63/99, FBColl-199/00, FBColl-244/00, FBColl- } \\
\text { 59/99, FBColl-95/99, FBColl-58/99, FBColl-65/99, FBColl-257/00, FBColl-269/00 }\end{array}$ \\
\hline $\mathrm{C}_{3}$ & 38 & $\begin{array}{l}\text { FBColl-146/99, FBColl-152/99, FBColl-170/99, FBColl-157/99, FBColl-134/99, FBColl-168/99, FBColl-138/99, } \\
\text { FBColl-137/99, FBColl-147/99, FBColl-161/99, FBColl-160/99, FBColl-133/99, FBColl-135/99, FBColl-148/99, } \\
\text { FBColl-156/99, FBColl-6/99, FBColl-74/99, FBColl-171/99, FBColl-150/99, FBColl-163/99, FBColl-139/99, } \\
\text { FBColl-169/99, FBColl-145/99, FBColl-144/99, FBColl-151/99, FBColl-142/99, FBColl-162/99, FBColl-176/00, } \\
\text { FBColl-261/00, FBColl-259/00, FBColl-26/99, FBColl-143/99, FBColl-165/99, FBColl-140/99, FBColl-172/99, } \\
\text { FBColl-159/99, FBColl-167/99, FBColl-23/99 }\end{array}$ \\
\hline $\mathrm{C}_{4}$ & 5 & FBColl-60/99, FBColl-153/99, FBColl-87/99, FBColl-173/99, FBColl-29/99 \\
\hline $\mathrm{C}_{5}$ & 18 & $\begin{array}{l}\text { FBColl-214/00, FBColl-217/00, FBColl-224/00, FBColl-226/00, FBColl-231/00, FBColl-233/00, FBColl-208/00, } \\
\text { FBColl-222/00, FBColl-209/00, FBColl-228/00, FBColl-211/00, FBColl-242/00, FBColl-205/00, FBColl-170/00, } \\
\text { FBColl-191/00, FBColl-201/99, FBColl-248/00, FBColl-246/00 }\end{array}$ \\
\hline $\mathrm{C}_{6}$ & 17 & $\begin{array}{l}\text { FBColl-157/00, FBColl-159/00, FBColl-161/00, FBColl-163/00, FBColl-164/00, FBColl-168/00, FBColl-172/00, } \\
\text { FBColl-178/00, FBColl-181/00, FBColl-184/00, FBColl-187/00, FBColl-188/00, FBColl-220/00, FBColl-236/00, } \\
\text { FBColl-255/00, FBColl-265/00, FBColl-267/00 }\end{array}$ \\
\hline $\mathrm{C}_{7}$ & 1 & FBColl-164/99 \\
\hline $\mathrm{C}_{8}$ & 1 & FBColl-204/00 \\
\hline
\end{tabular}




\begin{tabular}{ccc}
\hline Cluster & $\begin{array}{l}\text { No. of accessions } \\
\text { in cluster }\end{array}$ & Designations \\
\hline $\mathrm{C}_{1}$ & 66 & FPColl-56/00, FPColl-67/00, FPColl-202/99, FPColl-57/00, FPColl-83/00, FPColl-74/00, FPColl-44/99, FPColl-186/99, \\
& & FPColl-66/00, FPColl-58/00, \\
& FPColl-60/00, FPColl-65/00,FPColl-82/00, FPColl-209/99, FPColl-63/99, FPColl-189/99, FPColl-198/99, FPColl-195/99, \\
& & FPColl-207/99, FPColl-77/00, FPColl-68/00, FPColl-193/99, FPColl-184/99, FPColl-59/00, FPColl-61/00, FPColl-52/99, \\
& & FPColl-81/00, FPColl-54/99, FPColl-75/00, FPColl-71/00, FPColl-53/99, FPColl-194/99, FPColl-78/00, FPColl-196/99, \\
& FPColl-212/99, FPColl-37/99, FPColl-45/99, FPColl-36/99, FPColl-41/99, PColl-214/99, FPColl-215/99, FPColl-182/99, \\
& FPColl-42/99, FPColl-188/99, FPColl-76/00, FPColl-80/00, FPColl-73/00, FPColl-213/99, FPColl-45/00, PColl-72/00, \\
& FPColl-38/99, FPColl-51/99, FPColl-50/99, FPColl-79/00, FPColl-55/99, FPColl-197/99, FPColl-203/99, FPColl-210/99, \\
& FPColl-190/99, FPColl-206/99, FPColl-208/99, FPColl-57/99, FPColl-69/00, FPColl-185/99, FPColl-53/00
\end{tabular}

$\mathrm{C}_{2} \quad 50$

FPColl-30/99, FPColl-31/99, FPColl-32/99, FPColl-33/99, FPColl-34/99, FPColl-35/99, FPColl-39/99, FPColl-40/99, FPColl-43/99, FPColl-47/99, FPColl-48/99, FPColl-49/99, FPColl-127/99, FPColl-130/99, FPColl-187/99, FPColl-191/99, FPColl-192/99, FPColl-200/99, FPColl-201/99, FPColl-204/99, FPColl-205/99, FPColl-211/99, FPColl-216/99, FPColl217/99, FPColl-30/00, FPColl-31/00, FPColl-32/00, FPColl-33/00, FPColl-34/00, FPColl-35/00, FPColl-36/00, FPColl37/00, FPColl-38/00, FPColl-39/00, FPColl-40/00, FPColl-41/00, FPColl-42/00, FPColl-43/00, FPColl-44/00, FPColl46/00, FPColl-47/00, FPColl-48/00, FPColl-50/00, FPColl-51/00, FPColl-52/00, FPColl-54/00, FPColl-55/00, FPColl62/00, FPColl-64/00, FPColl-70/00, FPColl-84/00

FPColl-103/99, FPColl-106/99, FPColl-101/99, FPColl-104/99, FPColl-102/99, FPColl-100/99, FPColl-128/99, FPColl119/99, FPColl-125/99, FPColl-107/99, FPColl-112/99, FPColl-99/99, FPColl-98/99, FPColl-122/99, FPColl-115/99, FPColl-105/99, FPColl-132/99, FPColl-129/99, FPColl-131/99, FPColl-120/99, FPColl-126/99, FPColl-116/99, FPColl117/99, FPColl-111/99, FPColl-121/99, FPColl-108/99, FPColl-49/00, FPColl-114/99, FPColl-110/99, FPColl-123/99 
Mahalanobis's $\mathrm{D}^{2}$ analysis revealed that there was high genetic diversity among the Ethiopian faba bean and field pea accessions from different origins across the eco-geographic regions. Significant inter-cluster distances were recorded between most of the clusters, different members within a cluster being assumed to be more closely related in terms of the traits under consideration than those members in different clusters.
The diverse agro-climatic conditions in Ethiopia might have contributed to this genetic diversity in Ethiopian faba bean and field pea accessions as suggested by Harlan (1969). Members in clusters with non-significant distances were assumed to have more close relationships with each other than they do with those in significantly distant clusters (Tables $3 \mathrm{a}$ and $\mathrm{b}$, and Figures $3 \mathrm{a}$ and $\mathrm{b}$ ).

Table 3a. Pair wise generalized squared distances $\left(\mathrm{D}^{2}\right)$ among 160 faba bean accessions in eight clusters

\begin{tabular}{lcccccccc}
\hline Cluster & $\mathrm{C}_{1}$ & $\mathrm{C}_{2}$ & $\mathrm{C}_{3}$ & $\mathrm{C}_{4}$ & $\mathrm{C}_{5}$ & $\mathrm{C}_{6}$ & $\mathrm{C}_{7}$ & $\mathrm{C}_{8}$ \\
\hline $\mathrm{C}_{1}$ & 0 & $41^{* *}$ & 10 & $288^{* * *}$ & $53^{* * *}$ & $41^{* *}$ & $40^{* *}$ & $84^{* * *}$ \\
$\mathrm{C}_{2}$ & & 0 & 19 & $281^{* *}$ & 13 & $21^{* *}$ & $50^{* *}$ & $71^{* *}$ \\
$\mathrm{C}_{3}$ & & & 0 & $277^{* *}$ & $34^{* *}$ & $31^{* *}$ & $24^{*}$ & $75^{* *}$ \\
$\mathrm{C}_{4}$ & & & & 0 & $313^{* *}$ & $292^{* *}$ & $277^{* *}$ & $310^{* *}$ \\
$\mathrm{C}_{5}$ & & & & & 0 & $21^{*}$ & $77^{* *}$ & $35^{* *}$ \\
$\mathrm{C}_{6}$ & & & & & & 0 & $74^{* *}$ & $70^{* *}$ \\
$\mathrm{C}_{7}$ & & & & & & & 0 & $120^{* *}$ \\
$\mathrm{C}_{8}$ & & & & & & & & 0 \\
\hline
\end{tabular}

**Indicates highly significant difference at $p<0.01$

Table 3b. Pair wise generalized squared distances $\left(\mathrm{D}^{2}\right)$ among 148 field pea accessions in five clusters

\begin{tabular}{lccccc}
\hline Cluster & $\mathrm{C}_{1}$ & $\mathrm{C}_{2}$ & $\mathrm{C}_{3}$ & $\mathrm{C}_{4}$ & $\mathrm{C}_{5}$ \\
\hline $\mathrm{C}_{1}$ & 0 & 16 & $77^{* *}$ & $46^{* *}$ & $1046^{* *}$ \\
$\mathrm{C}_{2}$ & & 0 & $28^{* *}$ & $70^{* *}$ & $97^{* *}$ \\
$\mathrm{C}_{3}$ & & 0 & $146^{* *}$ & $1004^{* * *}$ \\
$\mathrm{C}_{4}$ & & & 0 & $1148^{* *}$ \\
$\mathrm{C}_{5}$ & & & & 0 \\
\hline
\end{tabular}

**Indicates highly significant difference at $p<0.01$

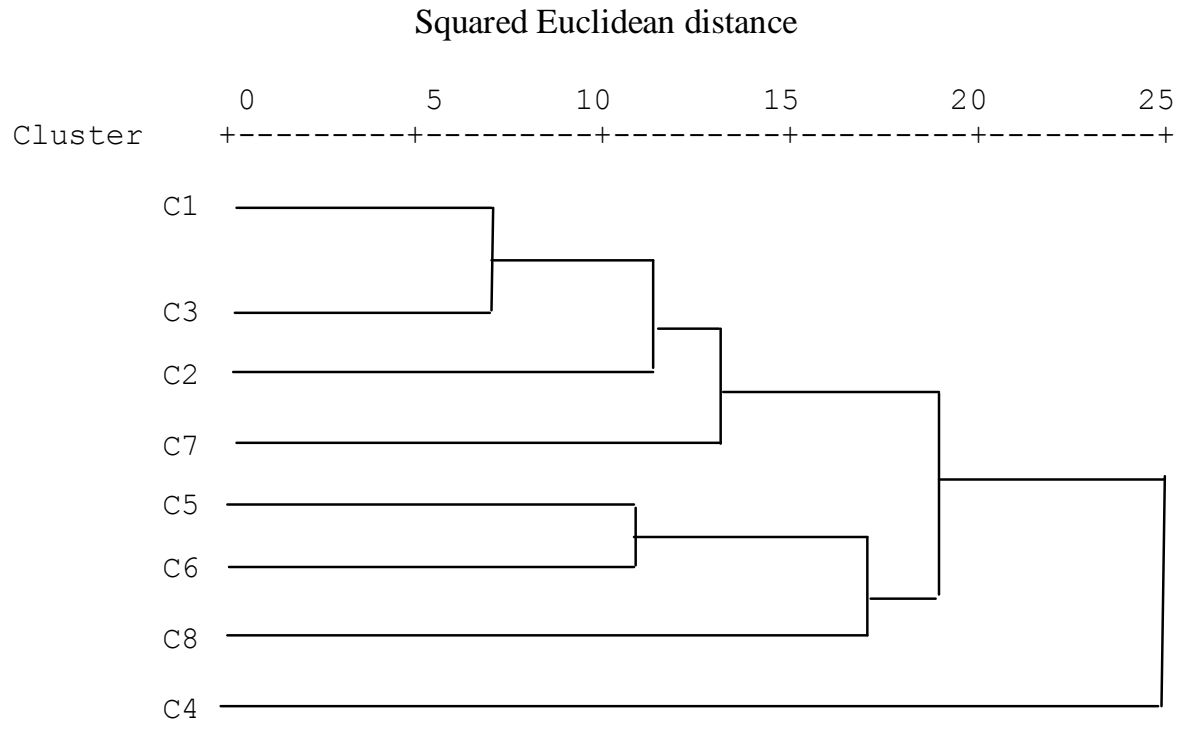

Figure 3a. Phenogram of faba bean accessions in seven clusters based on average linkage hierarchical cluster analysis between groups 


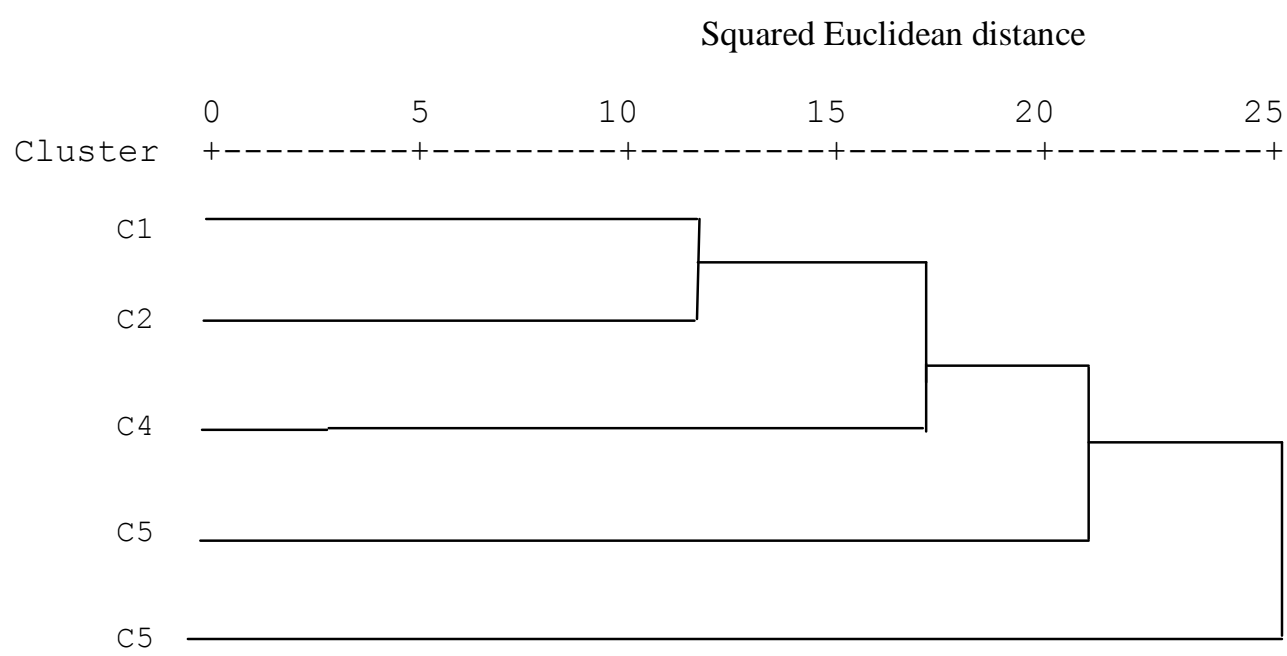

Figure 3b. Phenogram of field pea accessions in five clusters based on average linkage hierarchical cluster analysis between groups

The magnitude of genetic diversity and the relative contribution of the different morpho-agronomic traits to the differentiations of the accessions into clusters have been discussed elsewhere in detail (Gemechu et al., 2005 a,b) with only some difference due to pre-standardization of the data in this case.

\subsection{Eco-geographic Distribution of Genetic Diversity}

The distribution of strains in different clusters did not follow definite pattern with regard to geographical origins. However, accessions from the northern parts of the country (Wello and Gonder) tended to be grouped together for both crops showing their close interrelationships regardless of their geographic origin and the rugged nature of the terrain. This was clearly revealed from the fact that their distribution was limited to only three clusters for faba bean and two clusters for field pea (Tables $4 \mathrm{a}$ and $\mathrm{b}$ ).

Table 4a. Clustering pattern of 160 faba bean accessions collected from different eco-geographic regions of Ethiopia

\begin{tabular}{|c|c|c|c|c|c|}
\hline \multirow[t]{2}{*}{ Cluster } & \multirow{2}{*}{$\begin{array}{l}\text { No. of } \\
\text { accessions }\end{array}$} & \multicolumn{4}{|c|}{ Geographical distribution } \\
\hline & & Wello & Gonder & Shewa & Arsi \\
\hline 1 & 30 & 25 & --- & 5 & --- \\
\hline 2 & 50 & --- & 37 & 2 & 11 \\
\hline 3 & 38 & 3 & 1 & 31 & 3 \\
\hline 4 & 5 & 1 & 2 & 2 & --- \\
\hline 5 & 18 & --- & --- & --- & 18 \\
\hline 6 & 17 & --- & --- & --- & 17 \\
\hline 7 & 1 & --- & --- & 1 & --- \\
\hline 8 & 1 & --- & --- & --- & 1 \\
\hline
\end{tabular}

Table 4b. Clustering pattern of 148 field pea accessions collected from different eco-geographic regions of Ethiopia

\begin{tabular}{|l|l|r|r|r|r|}
\hline \multirow{2}{*}{ Cluster } & No. of & \multicolumn{4}{|c|}{ Geographical distribution } \\
\cline { 3 - 6 } & accessions & Wello & Gonder & Shewa & Arsi \\
\hline 1 & 66 & 15 & --- & 24 & 27 \\
\hline 2 & 50 & 11 & 2 & 10 & 27 \\
\hline 3 & 30 & --- & 29 & --- & 1 \\
\hline 4 & 1 & --- & --- & 1 & --- \\
\hline 5 & 1 & --- & --- & 1 & - \\
\hline
\end{tabular}


Populations from areas geographically far-separated with mountains and valleys, and having complex environments and varied ecological conditions are normally expected to accumulate enormous genetic diversity (Chandel and Joshi, 1983; Ford-Lloyd and Jackson, 1986; Singh, 2002) as geographical separation with physical barriers and genetic barriers to inter-crossing are believed to give rise to genetic diversity among genetic materials (Singh, 2002). Previous workers also proved that genetic diversity in accessions of both crops are not uniformly distributed across eco-geographic regions (Singh and Tripathi, 1985; Hailu et al., 1991; Dawit et al., 1994).

On the other hand, accessions from the southern part of the country (Arsi and Shewa) were distributed over most of the clusters but in an irregular pattern (Tables $4 \mathrm{a}$ and b). This indicates that, unlike in the northern parts of the country, there might be more intra-regional genetic diversity in the southern parts. This finding does not concur with the early estimate that more genetic diversity in these crops might rather be located in the northern half of the country (Hailu et al., 1991).

Clustering sites of faba bean and field pea collections at district levels discriminated them into five distinct broad clusters in both cases. Of the five clusters of faba bean collection sites, two $\left(\mathrm{C}_{2}\right.$ and $\left.\mathrm{C}_{5}\right)$ were constituted mostly from districts of Arsi with only a few exceptions. Districts from other parts did not produce a definite pattern of discrimination as different districts from the northern and southern parts of the country were grouped within the same clusters (Figures 4a). Similarly, classification of sites of field pea collections did not produce a definite pattern of discrimination as different districts from the northern and southern parts of the country were grouped within the same clusters (Figures 4b). This may indicate that distribution pattern of genetic diversity in faba bean was more influenced by geographic position than that of field pea.

Even though accessions from the northern parts of the country and those from the southern parts showed different genetic background in most of the cases, a few accessions from the two parts fell into the same clusters. This indicates that accessions from different regions may share similar genetic background. For instance, the third cluster in faba bean and the second in field pea constituted accessions from all regions. Similarly, the second and the first clusters in faba bean and field pea, respectively, comprised accessions from all regions except the absence of those from Gonder. Therefore, accessions may exhibit morpho-agronomic similarities regardless of differences in eco-geographic origins. Several possible reasons could be given for the genetic similarity among a few accessions from different corners of the country. It could be due to informal seed exchange among farmers in different ecogeographic regions of the country or a few materials might have originally been introduced from the same sources. There could also be a tendency (particularly among resource-poor farmers in marginal areas) of selecting for the same traits of interest like yield stability, resistance to diseases, insects and abiotic calamities and low dependence on the external inputs (de Boef et al., 1996).

\subsection{Microcenters of Genetic Diversity}

The higher genetic diversity in collections from Arsi and Shewa in both crops may indicate that the microcenter of genetic diversity for both crops may stretch somewhere from Arsi to Shewa. However, the reason for the higher genetic diversity in accessions collected from the southern part of the country as opposed to the ones from the northern parts of the country is not clear from this study. Differences in geographic origin appeared not to be the main cause of genetic divergence in both crops. This different pattern of diversity is probably attributed to the differences in the nature of both human and natural actions across regions as the genetic architecture of a population is generally believed to be the result of breeding system, gene flow within and between populations, isolation mechanisms and prolonged selection by various natural and artificial forces (Chandel and Joshi, 1983). There could be a tendency of selecting for the same traits of interest among farmers in the northern parts of the country as opposed to selection for different traits in the southern part that might have forced the crops to evolve in different directions. The original introduction to southern part of the country might have higher genetic differences compared to those introduced to the northern parts of the country. The nature of eco-geographic environment is also believed to be the major force in crop evolution (Ford-Lloyd and Jackson, 1986; Spagnoletti and Qualset, 1987). Rainfall distribution is the most variable elements of climate between the northern and the southern parts of Ethiopia (EMA, 1988; Asfaw et al., 1994 a,b) and suitable zones of faba bean and field pea production follow the pattern of rainfall distribution (Asfaw et al., 1994 a,b). It is believed that plants of a species growing in different environments may show different levels of diversity and that genetic diversity is higher under more suitable environments (Singh, 2002). Whether or not the lower level of genetic diversity in the northern part of the country has something to do with the recurrent drought in these parts of the country is not clear from this study. A systematic study with representative samples drawn from pre- and post-drought collections in this part of the country may enable to come up with conclusive results in this aspect. Similar results showing higher genetic diversity in accessions from Arsi as compared to those from Welo and Gojam were reported in barley (Demissie and Bjфrnstad, 1997). 


\section{Squared Euclidean distance}

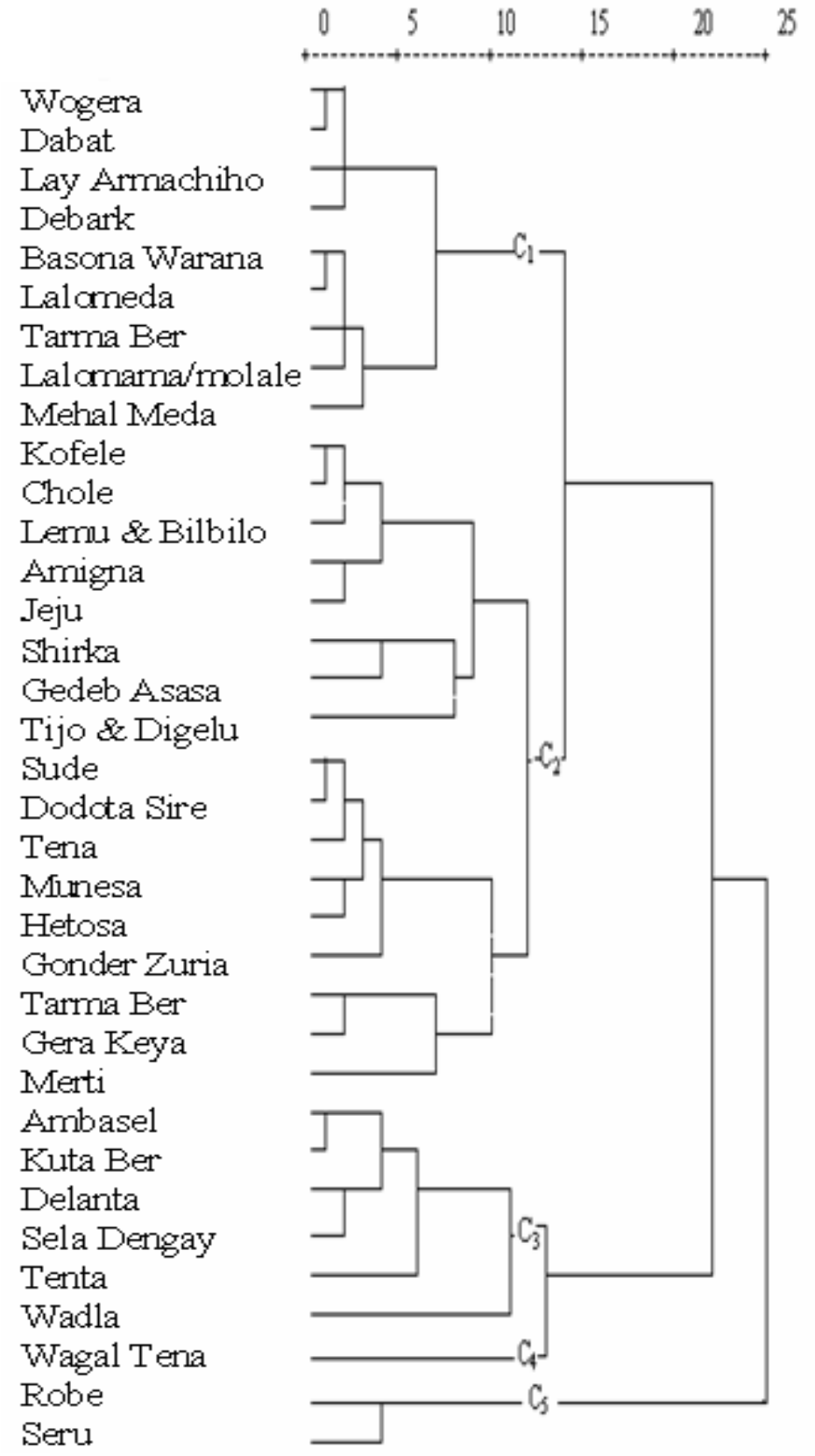

Figure 4a. Dendrogram of collection sites of faba bean accessions based on average linkage hierarchical cluster analysis between groups 
Squared Euclidean distance

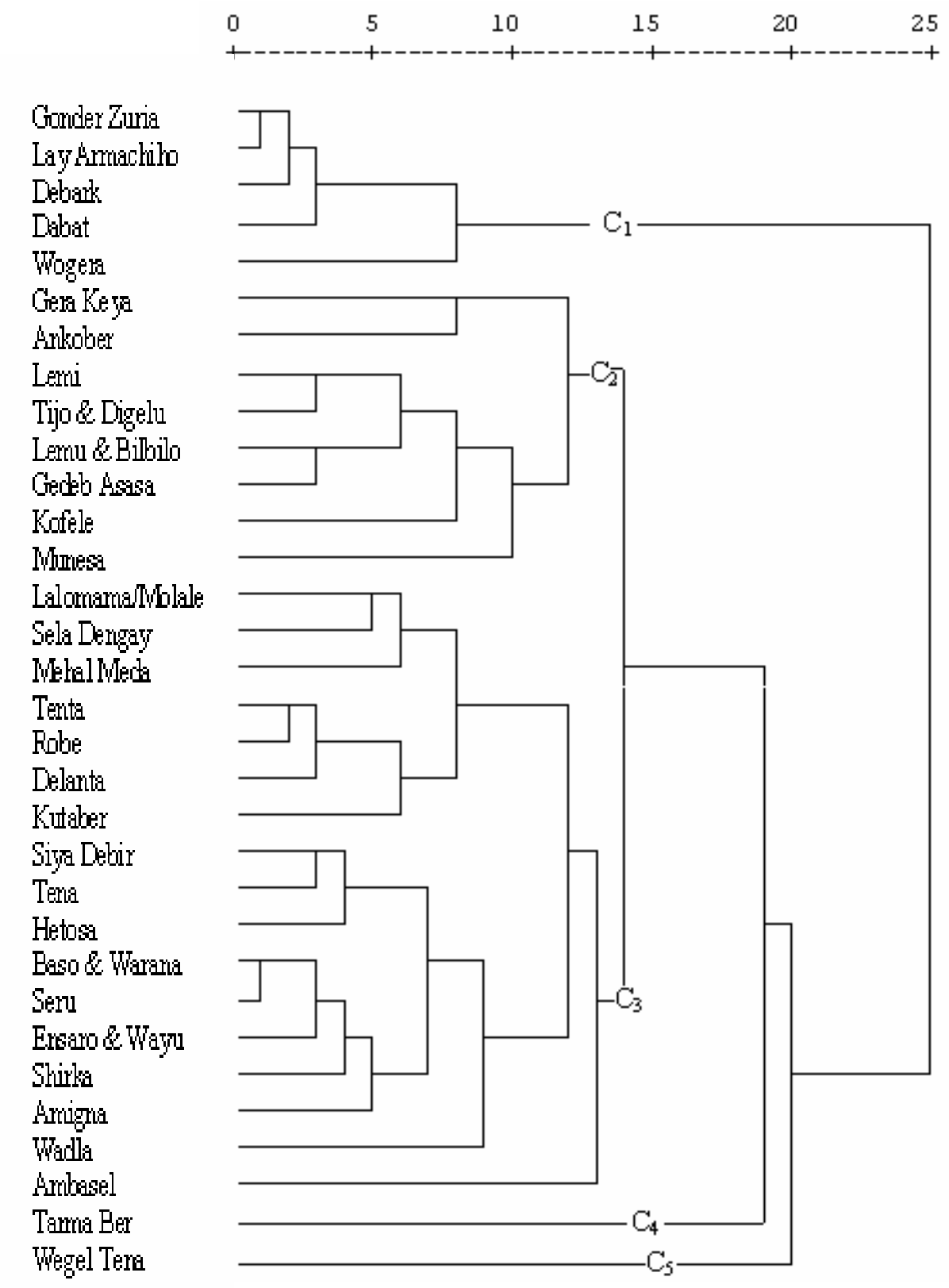

Figure 4b. Dendrogram of collection sites of field pea accessions based on average linkage hierarchical cluster analysis between groups 



\section{Conclusions}

The present findings clearly illustrated the existance of high genetic diversity in the Ethiopian faba bean and field pea accessions even though the diversity is not uniformly distributed across the eco-geographical regions. Germplsasm accessions of both crops from the northern parts of the country were closely related regardless of their geographic origin while those from southern parts were more diverse. This indicates that, unlike in the northern parts of the country, there might be more intra-regional genetic diversity in southern parts where the microcenters of diversity for both crops are probably located. The implication is that accessions from the northern parts of the country can be represented by a few samples whereas larger number of samples is needed to represent accessions from the southern parts of the country in order to catch most of the genetic variability.

The reason for differences in the distribution of genetic diversity in accessions between the northern and the southern parts of the country could not be clearly ascertained from this study. It could probably be related to the level of genetic diversity in the original introductions, and the nature and degree of natural and artificial manipulations after introductions may vary among regions. Future collection missions and conservation strategies should prioritize the southern parts of the country to safeguard the tremendous genetic diversity from genetic erosion, and ensure the sustainable perpetuation of these valuable resources. Breeding programs should also focus on effective and efficient exploitation of intra-regional diversity in this part. However, this does not mean that the whole area of genetic diversity and the peripheral regions should not be considered for the collection and conservation of accessions. Important rare and critical genes may sometimes exist in the peripheral regions (Ford-Lloyd and Jackson, 1986; Singh, 2002) as the crops could be exposed to different environmental stresses that may result in different lines of evolution (Singh, 2002). As a result, covering peripheral regions may enable capture rare genes associated with adaptation to different agroecologies. However, it should be noted that this investigation could provide only preliminary information, as a morpho-agronomic study alone may not be sufficient. Therefore, a comprehensive study involving both morpho-agronomic trait and molecular markers is required to draw a more comprehensive conclusion. In addition, a similar further study on representative samples from different districts in Arsi and Shewa may help come up with the accurate location of the microcenter of genetic diversity for both crops.

\section{Acknowledgments}

We appreciate the assistance of the Institute of Biodiversity Conservation (IBC), and Adet, Sheno and Sirinka Agricultural Research Centers in germplasm collection. We also wish to thank Mr. Nigusie Haile for making the maps with GIS and the staff of the Highland
Pulses Research Program at Holetta and Kulumsa Agricultural Research Centers for the field trial management and data collection.

\section{References}

Asfaw, T., Tesfaye, G. and Beyene, D. 1994a. Genetics and breeding of faba bean. In: Asfaw, T., Geletu, B., Saxena, M.C., and Solh, M.B. (eds.). CoolSeason Food Legumes of Ethiopia. Proceeding of the First National Cool-Season Food Legumes Review Conference, 16-20 December 1993, Addis Ababa, Ethiopia. ICARDA/IAR. ICARDA, Syria. pp.97-121.

Asfaw, T., Beyene, D. and Tesfaye, G. 1994b. Genetics and breeding of field pea. In: Asfaw, T., Geletu, B., Saxena, M.C., and Solh, M.B. (eds.). CoolSeason Food Legumes of Ethiopia. Proceeding of the First national Cool-Season Food Legumes Review Conference, 16-20 December 1993, Addis Ababa, Ethiopia. ICARDA/IAR. ICARDA, Syria. pp. 122-137.

Atta-Krah, K. 2004. Natural resources management and genetic diversity: Two sides of coin for sustainable livelihoods and development. A Paper Presented to the African Highland Initiative Conference - Integrated Natural Resource Management in Practice: Enabling Communities to Improve Mountain Landscapes and Livelihoods. 12-15 October 2004, Nairobi, Kenya (in press).

Bartual, R., Carbonell, E.A. and Green, D.E., 1985. Multivariate analysis of a collection of soybean cultivars for southeastern Spain. Euphytica 34:113-123.

Chahal, G.S. and Gosal, S.S.. 2002. Principles and Procedures of Plant Breeding: Biotechnological and Conventional Approaches. Narosa Publishing House, New Delhi.

Chandel, K.P.S. and Joshi, B.S. 1983. Multivariate analysis in green-seeded pea. Indian Journal of Agricultural Sciences 53 (4): 198-200.

Dale, M.F.B., Ford-Lloyd, B.V. and Arnold, M.H. 1985.Variation in some agronomically important characters in a germplasm collection of beet (Beta vulgaris L.). Euphytica 34: 449-455.

Davies, D.R. 1976. Peas: In: Simmonds, N.W. (ed.). Evolution of Crop Plants. Longman, London. pp. 172-174

Dawit, T., Asfaw, T. and Geletu, B. 1994. Accessions in Ethiopia. In: Asfaw, T., Geletu, B., Saxena, M.C., and Solh, M.B. (eds.). Cool-Season Food Legumes of Ethiopia. Proceeding of the First National Cool-Season Food Legumes Review Conference, 16-20 December 1993, Addis Ababa, Ethiopia. ICARDA/IAR. ICARDA, Syria. pp. 7996.

de Boef, W.S., Berg, T. and Haverkort, B. 1996. Crop accessions. In: Bunders J., Haverkort, B. and 
Hiemstra, W. (eds). Biotechnology: Building on Farmers' Knowledge. Macmillan, London and Basingstoke. pp. 103-128.

Demissie, A. and Bjфrnstad, A. 1997. Geographical, altitude and agro-ecological differentiation of isozyme and hordein genotypes of landrace barleys from Ethiopia: implications to germplasm conservation. Genetic Resources and Crop Evolution 44: 43-55.

EMA. 1988. National Atlas of Ethiopia. Ethiopian Mapping Authority (EMA), Addis Abeba.

Engels, J.M.M. and Hawkes, J.G. 1991. The Ethiopian gene center and its genetic diversity. In: Engels, J.M.M., Hawkes, J.G. and Melaku, W. (Eds.). Plant Genetic Resources of Ethiopia. Cambridge University Press. pp 23-41.

Ethiopian Agricultural Sample Enumeration. 2002. Report on the preliminary results of area, production and yield of temporary crops (meher season, private peasant holdings). Part I. Central Statistics Authority, Addis Ababa, Ethiopia.

FAO (Food and Agriculture Organization). 1998. The State of World Plant Accessions for Food and Agriculture. Rome, Italy.

Ford-Lloyd, B. and Jackson, M. 1986. Plant Accessions: An Introduction to Their Conservation and Use. Edward Arnold, Australia.

Gemechu, K., Mussa, J., Tezera, W. and Getnet, D. 2005a. Extent and pattern of genetic diversity for morpho-agronomic traits in Ethiopian highland pulse landraces. I. Field pea (Pisum sativum L.). Genetic Resources and Crop Evolution 52: 539-549.

Gemechu, K., Mussa, J., Tezera, W. and Getnet, D. 2005b. Extent and pattern of genetic diversity for morpho-agronomic traits in Ethiopian highland pulse landraces. II. faba bean (Vicia faba L.). Genetic Resources and Crop Evolution 52: 551-561.

Hagedorn, D.J. 1984. Compendium of Pea Diseases. University of Wisconsin-Madison.

Hailu, M., Abebe, D. and Abebe, T. 1991. Pulse crops of Ethiopia. In: Engels, J.M.M., Hawkes J.G. and Melaku, W. (Eds.). Plant Genetic resources of Ethiopia. Cambridge University Press.pp328-343.

Harlan, J.R. 1969. Ethiopia: A Center of Diversity. Economic Botany 23: 309-314.

Jaradat, A.A. 1991. Phenotypic divergence for morphological and yield related traits among landrace genotypes of durum wheat from Jordan. Euphytica 52:155-164.

Little, T.M. and Hills, F.J. 1978. Agricultural Experimentation: Design and Analysis. John Wiley and Sons. New York
Manly, B. F. J. 1986. Multivariate Statistical Methods: A Primer. Chapman and Hall. London.

Nigussie, A. 2001. Germplasm diversity and genetics of quality and agronomic traits in Ethiopian mustard (Brassica carinata A. Braun). Doctoral dissertation, Georg-August University of Göttingen, Germany.

Rezai, A. and Frey, K.J., 1990. Multivariate analysis of variation among wild oat accessions-seed traits. Euphytica 49:111-119.

SAS Institute. 1996. SAS/STAT guide for personal computers, version 6.12 edition. Cary, NC: SAS Institute Inc.

Singh, R.K. and Chaudhary, B.D. 1985. Biometrical Methods in Quantitative Genetic Analysis. Kalyani Publishers, New Delhi-Ludhiana.

Singh, S.B. and Tripathi, B.K.. 1985. Genetic divergence in pea. Indian Journal of Genetics 45: 389-393.

Singh, B.D. 2002. Plant Breeding: Principles and Methods. Kalyani Publishers, New DelhiLudhiana.

Spagnoletti, P.L. and Qualset, C.O. 1987. Geographical diversity for quantitative spike characters in a world collection of durum wheat. Crop Science 27: 235-241.

Tesema, T. and Eshetayehu, T. 2004. Collection, conservation, characterization and sustainable utilization of grain legumes in Ethiopia. A paper presented to the $2^{\text {nd }}$ National Workshop on Food and Forage Legumes in Ethiopia, 22-26 Sept. 2003, Addis Ababa, Ethiopia (in press).

Van der Maesen, J.J.G, Kaiser, W.J., Marx, G.A. and Worede, M. 1988. Genetic bases for pulse crop improvement: Collection, preservation and genetic variation in relation to need traits. In: Summerfield (ed.), Proceedings of the International Food Legume Research Conference on Pea, Lentil, Faba Bean, Chickpea, Spokane, Washington, USA, 6-11 July, 1986. Kluwer, Dordrecht. PP. 5566.

Vavilov, N.I. 1951. The Origin, Variation, Immunity and Breeding of Cultivated Plants. Chronica Botanica, Waltham, Mass, USA.

Westphal, E. 1974. Pulses in Ethiopia: Their Taxonomy and Significance. College of Agriculture, Haile Sellessie I University, Ethiopia/ Agriculture University, Wageningen, The Netherlands.

Yohannes, D. 2000. Faba bean (Vicia faba) in Ethiopia. Institute of Biodiversity Conservation and Research (IBCR). Addis Ababa, Ethiopia. 